\title{
3-D Multiscale Adaptive Eulerian-Lagrangian Method for Multiphase Flows with Phase Change
}

\author{
Jaeheon Sim*, Chih-Kuang Kuan* and Wei Shyy ${ }^{\dagger}$ \\ University of Michigan, Ann Arbor, MI, 48109
}

\begin{abstract}
A multi-scale multiphase computational model including phase change has been developed to study the moving interfacial dynamics and thermal effect in various engineering and scientific applications, including spacecraft cryogenic propellant delivery processes. A 3-D adaptive Eulerian-Lagrangian method is implemented, utilizing the stationary (Eulerian) frame to resolve the flow field, and the marker-based triangulated moving (Lagrangian) surface meshes to treat the fluid interface and solid boundaries. Other than treating the unsteady, convection, pressure, viscous/diffusion, and buoyancy terms in the governing field equations, the energy and mass transfer across interface due to phase change is handled using probe-based profile computations. Numerous test cases are presented, including liquid fuel draining, sloshing, and surface flow stability related to the interfacial dynamics, and natural convection in a cavity and Stefan problem for energy transport and phase change dynamics.
\end{abstract}

\section{Introduction}

$\mathrm{W}$ HEN designing and operating a spacecraft, the dynamics and managements of the cryogenic propellants in a spacecraft fuel tank is one of the major concerns. The draining and sloshing motion under microgravity have critical impact on the vehicle dynamics control due to the shift in its center of mass. Furthermore, the variation on the amount of fuel delivered to the combustion chamber may result in failure of engine operation and following mission failure. The heat transfer from the tank sidewall makes the phenomena more complicated since the thermal stratification and buoyancy have a significant influence on the distribution of liquid fuel and boiling of liquid fuel makes changes in fuel tank pressurization. These thermal stratification and pressurization may results in critical engine shutdown due to cavitation in the propellant pump. While understanding the flow motions and thermal effects in a liquid fuel tank and its influence on the spacecraft dynamics and fuel delivery are crucial for spacecraft applications, opportunities for experimental investigations are limited due to the requirements for test conditions such as large acceleration change from high- to micro-gravity.

Numerical simulations of interfacial flows can play instrumental roles in helping improve our capabilities of resolving these issues. The present effort is aimed at developing suitable computational modeling techniques capable of offering adequate resolution of moving interfacial dynamics, phase change and following mass transfer, and interactions between phase boundaries and complex solid boundaries. As reviewed in Shyy et al. (1996), three main categories exist in moving boundary computations, including Lagrangian (moving grid), Eulerian (stationary grid), and Eulerian-Lagrangian methods. ${ }^{1}$ Lagrangian methods are accurate due to its body-fitted grid, but computationally expensive and difficult when large deformation/movements and/or topological changes take place. The Eulerian (stationary grid) method extracts the interface location via post processing with the help of a scalar function on a stationary computational grid, and its simplicity and natural handling of topological change makes these methods very convenient. ${ }^{2,3}$ Eulerian-Lagrangian methods utilize a separate set of moving (Lagrangian) mesh and associated marker/tag system representing interface on a stationary (Eulerian) grid used to compute the flow fields. Thus, the interface can be tracked explicitly, and these methods possess several desirable features when compared with Lagrangian or Eulerian methods. ${ }^{4,5}$

The Eulerian-Lagrangian method has been widely used due to its explicit tracking of interface and relative simplicity. Peskin proposed immersed boundary method categorized into continuous interface method, which facilitates a single fluid formulation for the entire domain by smoothing out the fluid property and models the surface tension forces as a momentum source term within finite range instead of an interface of zero thickness. ${ }^{6}$

\footnotetext{
* Graduate Student, Department of Aerospace Engineering, Student member AIAA.

${ }^{\dagger}$ Clarence L. "Kelly" Johnson Collegiate Professor and Chair, Department of Aerospace Engineering, Fellow AIAA.
} 
These continuous interface method is implemented by many researchers to simulate large deformable or merger/breakup fluid flows. ${ }^{4,7}$ Mittal implemented a kind of sharp interface method with the help of ghost cell by reconstructing solution on the ghost cells to satisfy the jump condition between the flow fields on two sides of a phase boundary exactly on the interface of zero thickness. ${ }^{8}$ Cut-cell and ghost fluid method were also proposed in this category. Usually, fluid/solid interface is modeled by sharp interface method due to small deformation and no need to solve solid phase while fluid/fluid interface is modeled by continuous method because it's easier to implement and capable of handling various multiphase problems even with large deformation and moving interfaces. Eray and Jaeheon implement both continuous and sharp interface method together with adaptive Cartesian grid. ${ }^{9}$ The arbitrary solid boundary for practical engineering problem is represented by sharp interface method, and large-deformable fluid/fluid interface is modeled by continuous interface method.

The phase change and following mass transfer across phase boundary has not been known well due to its physical and numerical complexity. Juric and Tryggvason extended their immersed boundary method into boiling problem by adding smoothed latent heat source term into energy equation, and iterate the whole solution procedure estimating the amount of mass transfer. ${ }^{10}$ Shin and Juric simplifies the method computing the mass transfer from the known temperature gradient. ${ }^{11}$ However, diffused material properties like thermal conductivity limited the accuracy of mass computation. Luo et al. implemented sharp treatment for temperature computation with standard continuous level-set method. ${ }^{12}$ In this research, the sharp interface method is used for temperature fields with continuous treatment of velocity and pressure fields. The mass transfer is computed from temperature gradient using discontinuous material property, and the temperature is corrected around interface for better accuracy as Morgan did. ${ }^{13}$ The sharp treatment for temperature is known to produce more accurate results, but it is very difficult to implement this method with interface with large deformation. Ferziger showed an interesting study that the errors in continuous treatment may be from the incorrect smoothed average of thermal conductivity around interface in steady pure heat conduction problem with no phase change. ${ }^{14}$

In the present study, a 3-D adaptive Eulerian-Lagrangian method has been improved from the previous research ${ }^{15}$ especially on the thermal effect including phase change and solid boundary treatment with contact line and local slip model. It utilizes the stationary (Eulerian) frame to resolve the flow field, and the marker-based triangulated moving (Lagrangian) surface meshes to treat the phase boundary interfaces. The multiphase fluid boundaries are modeled using a continuous interface method, and the surface tension between fluid interfaces is smeared within finite distance. The solid boundaries are treated by a sharp interface method along with the ghost cell method by reconstructing the solution on the ghost cell based on the known solid boundary condition. The contact line where the fluid interface meets solid boundary is modeled using contact line force model, which enforces the given contact angle dynamically. For moving contact line treatment, local slip condition is applied around contact line. The adaptive Cartesian grid method is implemented to resolve the enough computation resolution especially around interface with effective computation. The energy equation solver is added into the previous study, and Boussinesq approximation is used for Buoyancy force computation. The phase change model using continuous treatment for temperature is developed with modified smoothing of material properties. The draining, sloshing, and surface stability of liquid fuel is summarized for the illustration of performance in solving moving interfacial dynamics problem. The natural convection in a cavity and one- and two-phase Stefan problem are tested and compared with benchmark solution and theoretical values for the validation of the present numerical approaches.

\section{Numerical Methods}

In the present study, the marker-based 3-D adaptive Eulerian-Lagrangian method is implemented to perform incompressible interfacial flow computations including phase change around irregular solid geometries. The bulk flow variables are solved on the stationary (Eulerian) background grid, whereas interface variables are handled by moving (Lagrangian) markers. The components of the numerical algorithm and their interactions are summarized in Figure 1, and detailed numerical method can be found in the previous works. ${ }^{7,9,15}$

\section{A. Governing equations}

A single fluid formulation for all fluid phases is made possible by smoothing out the properties across the interface. Non-dimensionalized incompressible Navier-Stokes equations for mass and momentum conservation are given in Eqs. (1) and (2) respectively, which accounts for the interfacial dynamics as source terms in the governing equations; surface tension effects of fluid interfaces as a momentum forcing term $\left(\mathbf{F}_{f}\right)$, and the latent heat effects across fluid interfaces as a energy source term $\left(Q_{f}\right)$. In Eqs. (1) and (2), $\mathbf{u}$ is the velocity vector, and $\rho, \mu$, and $p$ is the density, viscosity, and pressure, respectively. 


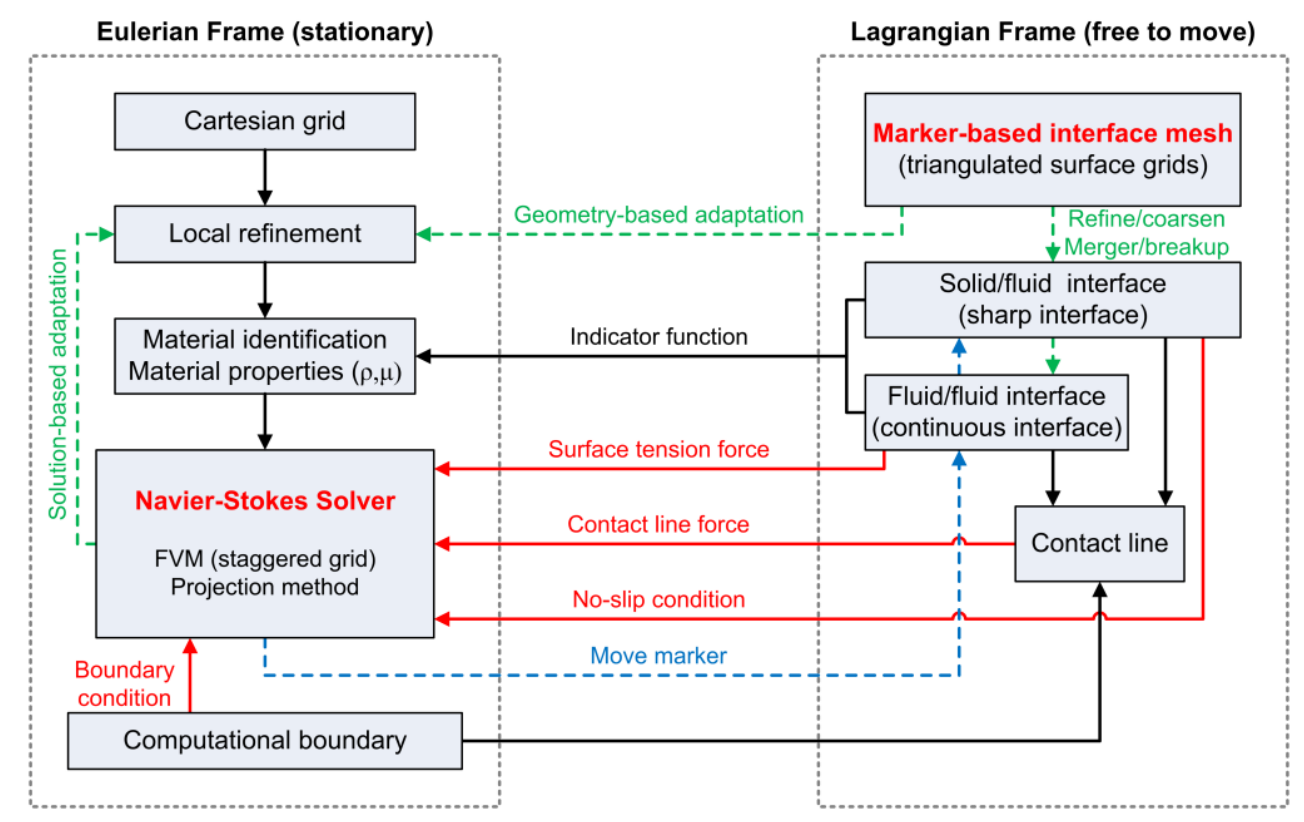

Figure 1. Summary of the numerical method and the interactions between Eulerian and Lagrangian descriptions.

$$
\begin{gathered}
\frac{\partial \rho}{\partial \mathrm{t}}+\boldsymbol{\nabla} \cdot(\rho \mathbf{u})=0 \\
\frac{\partial \rho \mathbf{u}}{\partial \mathrm{t}}+\boldsymbol{\nabla} \cdot(\rho \mathbf{u u})=-\nabla \mathrm{p}+\frac{1}{\mathrm{Re}} \boldsymbol{\nabla} \cdot\left(\mu \boldsymbol{\nabla} \mathbf{u}+\mu \boldsymbol{\nabla}^{\mathrm{T}} \mathbf{u}\right)+\frac{1}{\mathrm{Fr}} \rho \mathbf{g}+\frac{1}{\mathrm{We}} \mathbf{F}_{\mathrm{f}} \\
\frac{\partial \rho \mathrm{CT}}{\partial \mathrm{t}}+\boldsymbol{\nabla} \cdot(\rho \mathrm{CT} \mathbf{u})=\boldsymbol{\nabla} \cdot(\mathrm{K} \boldsymbol{\nabla T})+\mathrm{Q}_{\mathrm{f}}
\end{gathered}
$$

Here, all variables are non-dimensionalized by a characteristic velocity (U) and length scale (L), standard gravity $\left(\mathrm{g}_{0}\right)$, and liquid material properties (density $\rho_{\mathrm{l}}$, viscosity $\mu_{\mathrm{l}}$, and surface tension $\sigma$ ). The non-dimensional parameters of Reynolds, Froude, and Weber numbers in Eq. (2) are defined as, $\operatorname{Re}=\rho_{\mathrm{l}} \mathrm{UL} / \mu_{1}, \mathrm{Fr}=\mathrm{U}^{2} /\left(\mathrm{g}_{0} \mathrm{~L}\right)$, and We $=$ $\rho_{\mathrm{l}} \mathrm{U}^{2} \mathrm{~L} / \sigma$. In addition, the capillary number and bond number can be defined as $\mathrm{Ca}=\mathrm{We} / \mathrm{Re}=\mu_{\mathrm{l}} \mathrm{U} / \sigma$ and $\mathrm{Bo}=$ $\mathrm{We} / \mathrm{Fr}=\rho_{1} \mathrm{~g}_{0} \mathrm{~L}^{2} / \sigma$, respectively.

In order to facilitate a single set of equations formulation of a continuous interface method for the whole domain, a smooth variation of discontinuous material properties across interfaces is allowed. This is achieved with the help of indicator function (I), a scalar function varying from zero to one smoothly across interface and having 0.5 at the interface location. The smoothed fluid properties are computed using Eqs. (4)-(7).

$$
\begin{gathered}
\rho=\rho_{2}+\left(\rho_{1}-\rho_{2}\right) \mathrm{I} \\
\frac{\rho}{\mu}=\left(\frac{\rho}{\mu}\right)_{2}+\left[\left(\frac{\rho}{\mu}\right)_{1}-\left(\frac{\rho}{\mu}\right)_{2}\right] \mathrm{I} \\
\rho \mathrm{C}=(\rho \mathrm{C})_{2}+\left[(\rho \mathrm{C})_{1}-(\rho \mathrm{C})_{2}\right] \mathrm{I} \\
\frac{\rho \mathrm{C}}{\mathrm{k}}=\left(\frac{\rho \mathrm{C}}{\mathrm{k}}\right)_{2}+\left[\left(\frac{\rho \mathrm{C}}{\mathrm{k}}\right)_{1}-\left(\frac{\rho \mathrm{C}}{\mathrm{k}}\right)_{2}\right] \mathrm{I}
\end{gathered}
$$


Separate indicator functions for fluid and solid interface are used to separate the designation of solid interface, modeled by a sharp interface method, from fluid interface modeled by a continuous interface method. The indicator function is computed using a discrete form of the Heaviside step function in Eq. (8) by integrating 1-D form of discrete Dirac delta function. This approach is known being applicable more generally than Poisson equation solver method since it requires only distance information from interface, and thus gives accurate values even at the boundaries. $^{9}$

$$
\mathrm{I}(\mathbf{x})=\mathrm{H}(\mathrm{r}=\mathbf{n} \cdot(\mathbf{x}-\mathbf{X}))=\int_{-\infty}^{\mathrm{r}} \delta(\mathrm{h}) \mathrm{dh}
$$

The governing equations (1) and (2) are solved using a projection method with a staggered grid finite volume formulation. The convection term is discretized using 3rd order ENO scheme in space and 2nd order Runge-Kutta integration in time. The central difference scheme and Crank-Nicholson method is implemented for viscous term.

The indicator function in the Eulerian framework $(\mathbf{x})$ is computed from Lagrangian quantity $(\mathbf{X})$ with the help of a discrete Dirac delta function. The analytical form of the Dirac delta function is only non-zero at $\mathbf{x}=\mathbf{X}$, where the interface is located. However, this approach is difficult to implement along with a discretized set of equations as the discrete points on Eulerian and Lagrangian framework do not necessarily coincide. For this reason, an approximate Dirac delta function, originally proposed by Peskin, is implemented over finite thickness, 4 cell widths in the present study. ${ }^{6}$

\section{B. Interface representation and tracking}

In this marker-based Eulerian-Lagrangian method, the interface is represented by massless markers in coordination with each other for maintaining the interface connectivity information. The corresponding data structure is established via line-segments in twodimensional and triangles in three-dimensional domains, as represented in Figure 2(a). Markers store the surrounding elements' indices while elements store the neighboring elements based on the edge that they share. For boundary elements, negative value is used for referring computational boundary or intersecting solid elements in Figure 2(b).

The marker locations, denoted by $\mathbf{X}$ in Lagrangian frame, are updated from the velocities at its location, $\mathbf{V}(\mathbf{X})$, in Eq. (9).

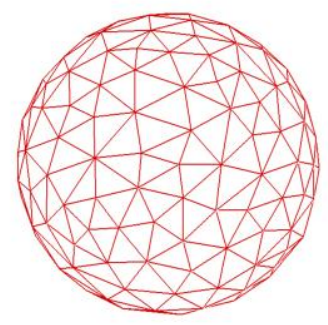

(a)

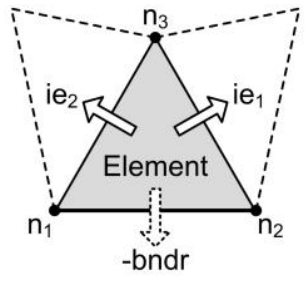

(b)

Figure 2. Interface representation (a) Interface surface represented by massless markers and triangular elements, (b) connectivity information for boundary triangular element with two neighboring elements.

$$
\frac{\partial \mathbf{X}}{\partial \mathrm{t}}=\mathbf{V}(\mathbf{X})
$$

Fluid interfaces use the computed flow solution field to obtain the marker velocities as shown in Eq. (10). In this equation, the discrete Dirac delta function, $\delta(\mathbf{x}-\mathbf{X})$, is employed for converting the Eulerian velocity field, $\mathbf{u}(\mathbf{x})$, to Lagrangian form, $\mathbf{U}(\mathbf{X})$. The interface velocity is exactly same to fluid velocity if there is no mass transfer in Eq. (10). However, with phase change, the velocity component from mass transfer should be considered. On the other hand, solid interfaces use the prescribed velocity field to advance the marker points using Eq. (9).

$$
\mathbf{U}(\mathbf{X})=\int_{\mathrm{v}} \mathbf{u}(\mathbf{x}) \delta(\mathbf{x}-\mathbf{X}) \mathrm{dV}-\frac{\dot{\mathrm{m}}}{\rho_{\mathrm{f}}} \cdot \mathbf{n}
$$

As the marker points advance to a new position with time, the interface surface mesh can exhibit an uneven distribution of marker points. In order to maintain consistent computational accuracy, the spacing between marker points is rearranged by adding or deleting markers whenever two markers come too close or too distant from each other. The volume errors during these processes can be recovered by adjusting the location of the modified marker in its normal direction, as discussed by Singh and Shyy. ${ }^{7}$

The level-contour-based interface reconstruction technique with connectivity information is implemented to handle topological changes such as merger or break-up. With connectivity information, the time-consuming 
reconstruction is facilitated only in case of merger/break-up by examining the possibility of topological changes at prescribed time intervals. These reconstruction procedures can be done locally for a specific interface body, and the errors in phase volume after reconstruction are explicitly corrected by perturbing the markers in local normal direction.

\section{Fluid interface treatment: continuous interface method}

The continuous interface method is implemented for all fluid phases by a single fluid formulation due to its effectiveness in modeling highly deformable fluid interfaces. The surface force computation of Eq. (11), where $\sigma$ is the surface tension and $\kappa$ is the curvature of interface, is applied as source term in momentum Eq. (2).

$$
\mathbf{F}_{\mathrm{f}}=\int_{\mathrm{A}} \sigma \kappa \delta(\mathbf{x}-\mathbf{X}) \mathrm{dA}
$$

The surface force of interface is also transformed from Lagrangian quantity $(\mathbf{X})$ to Eulerian quantity $(\mathbf{x})$ via the approximate discrete Dirac delta function, $\delta(\mathbf{x}-\mathbf{X})$. The computation on a discretized interface element (curves in 2D and triangles in 3D) is conducted by a line integral form shown in Eq. (12) with normal and tangent vectors instead of a direct curvature computation since the curvature computation using interpolation-based methods is numerically sensitive and often requires some form of data smoothing, and does not enforce surface tension conservation on a closed surface.

$$
\boldsymbol{\delta} \mathbf{f}=\int_{\delta \mathrm{A}} \sigma(\mathbf{n} \times \boldsymbol{\nabla}) \times \mathbf{n d A}=\int_{\mathrm{s}} \sigma(\mathbf{t} \otimes \mathbf{n}) \mathrm{ds}
$$

\section{Solid interface treatment: sharp interface method}

Solid interfaces is modeled using the sharp interface method by reconstructing solution fields around an interface to incorporate the given boundary condition on the solid interface in an Eulerian Cartesian grid. Solution reconstruction can be conducted either on the fluid side or solid side. Both approaches work well for a single phase flow around solid surfaces. However, the fluid velocity reconstruction method ignores the influence of surface tension from fluid interface by reconstructing fluid velocities directly, and thus, tend not to describe moving phase boundary near solid interface. Alternatively, the velocity reconstruction on the solid side can be implemented via ghost cells, which are defined as solid cells having at least one neighboring fluid cell. This approach works well even with contact line where fluid interface meets solid interface.

The solution reconstruction on the ghost cell is conducted by linear or bi/trilinear interpolation scheme

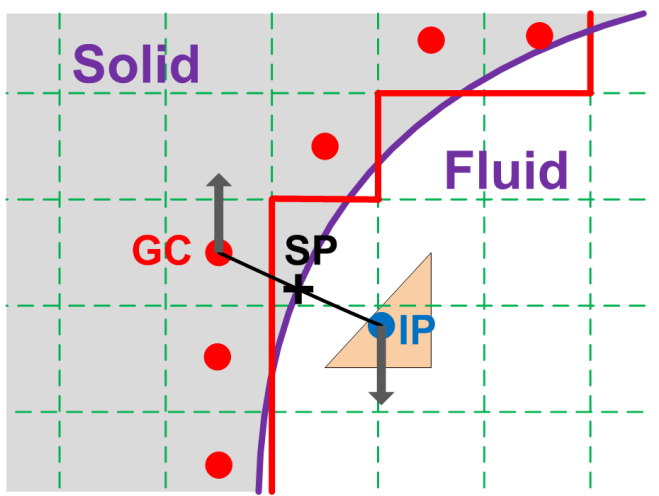

Figure 3. Identification of ghost cell (GC), solid point (SP), and imaginary point (IP). The interpolation of velocity is conducted on the imaginary point first, and then computed at the ghost cells based on the velocity of solid point. using solutions at the solid surface and at the known fluid cells. Avoiding extrapolation due to the location of ghost cell in solid phase, the interpolation can be conducted first at an imaginary point in fluid phase, and then, the solution of ghost cells can be obtained from the interpolated value at the imaginary point and a known value at a solid surface.

\section{E. Contact line treatment}

When we consider a fluid-fluid interface intersecting a solid surface, the treatment of the tri-junction locations, called contact line, is required to account for the presence and interactions of all three phases, fluid-fluid-solid. One of the most discussed issues for modeling these contact lines with Navier-Stokes equations is that the imposed noslip condition for velocity leads to a non-integrable singularity in stress. In the present research, the contact line force is imposed with local slip condition to overcome this singularity issue. 
In Figure 4, the angle at the contact line can be used for representing the balance of forces resulting from intermolecular forces between solid, liquid and gas phases. The tangential component of the resulting force at the contact line $\left(F_{R}\right)$ is shown in Eq. (13). In static equilibrium, Eq. (13) leads to the well-known Young-Laplace equation as given in Eq. (14).

$$
\begin{gathered}
\mathrm{F}_{\mathrm{R}}=\sigma_{\mathrm{sg}}-\sigma_{\mathrm{sl}}-\sigma \cos \theta \\
\sigma_{\mathrm{sg}}=\sigma_{\mathrm{sl}}+\sigma \cos \theta_{0}
\end{gathered}
$$

In Eq. (14), $\sigma_{\mathrm{sg}}$ is the surface force due to the interaction of

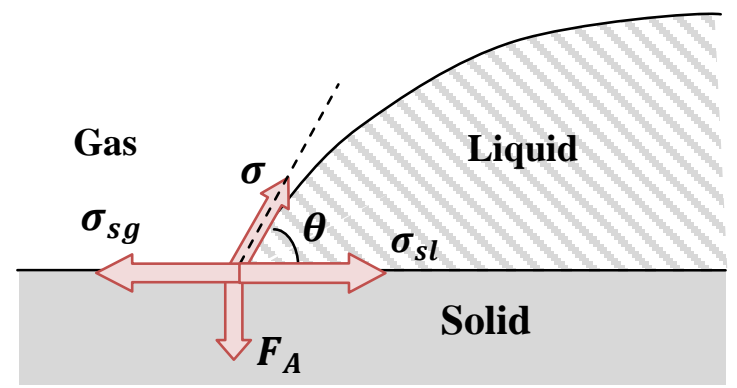

Figure 4. Forces at tri-junction (contact line). $F_{A}$ represents the adhesive forces.

solid and gas, $\sigma_{\mathrm{sl}}$ is the surface force due to the interaction of solid and liquid and $\sigma$ is the surface tension defined between liquid and gas. The given static contact angle is represented by $\theta_{0}$, and $\theta$ is the present contact angle at an instant. In this approach, the force at the contact line is obtained by plugging Eq. (14) into Eq. (13). This contact line force in Eq. (15) accelerates or decelerates flow fields, and makes the contact angle $(\theta)$ asymptotically towards a prescribed static contact angle $\left(\theta_{0}\right)$.

$$
\mathrm{F}_{\mathrm{R}}=\sigma\left(\cos \theta_{0}-\cos \theta\right)
$$

During the computation of the source term due to surface tension, the contact line region contributes as a recovery force in the tangential direction to the solid surface $\left(\mathbf{t}_{\mathbf{s}}\right)$ instead of the curvature effects given in Eq. (16). The contact angle is enforced on open edges where the element connects to either a computational boundary or a solid interface by modifying Eq. (12). The surface tension force from interface curvature is applied to closed edges, and the contact line force is applied to open boundary edges.

$$
\boldsymbol{\delta} \mathbf{f}=\sum_{\substack{\mathrm{i}=\text { closed } \\ \text { edges }}} \sigma\left(\mathbf{t}_{\mathbf{f}} \otimes \mathbf{n}\right)_{\mathrm{i}} \Delta \mathbf{s}+\sum_{\substack{\mathrm{i}=\text { open } \\ \text { edges }}} \sigma\left(\cos \theta_{0}-\cos \theta\right)_{\mathrm{i}} \mathbf{t}_{\mathbf{s}} \Delta \mathbf{s}
$$

The slip velocity condition on interface markers at the contact line is imposed during the transfer of Eulerian velocity field into the Lagrangian marker points. Instead of imposing the full Dirac function region in Eq. (10), we consider a one-sided averaging via Dirac function only for fluid side. This treatment is represented by Eq. (17).

$$
\mathbf{U}(\mathbf{X})=\frac{\sum_{\mathrm{x}} \mathbf{u}(\mathbf{x}) \delta(\mathbf{X}-\mathbf{x})}{\sum_{\mathrm{x}} \delta(\mathbf{X}-\mathbf{x})}
$$

In Eq. (17), the summation of delta function in denominator will yield unity (as a property of delta function) further away from the contact line whereas it is less than unity for markers in the proximity of a solid surface. As a result, casting the velocity field on the contact line yields a slip condition on the solid surface.

Local slip condition is applied around contact line on solid boundary to simulate the moving contact line problems with better accuracy. The present model and solid boundary treatment work well with no-slip condition for steady and/or slowly moving fluid interface problems. However, the no-slip condition imposed on the solid leads to a non-integrable singularity in stress and retarded interface movement due to the discontinuous velocity fields in Figure 5(a). This singularity can be solved by applying slip condition within finite distance from contact line using Dirac-delta function in Figure 5. A perfect slip is applied exactly on the contact line, and partial slip is within 2 cell distance as continuous fluid interface is

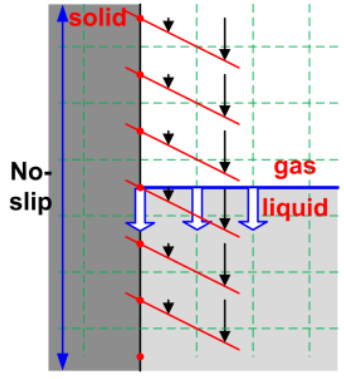

(a)

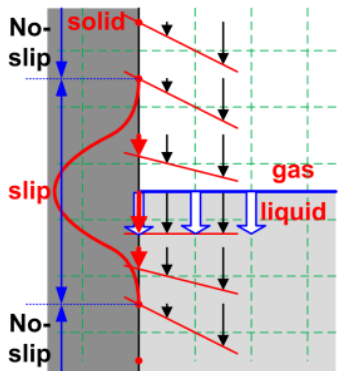

(b)

Figure 5. The comparison of velocity fields between no-slip and local slip condition. (a) no-slip condition (b) local slip application. 


\section{F. Adaptive Grid}

Multiphase flow problems involve multiple length scales. In order to effectively resolve the flow features in such cases, adaptive grid based on isotropic refinement is implemented. The cells are split into four and eight equal sibling cells in two- and three-dimensions, respectively to better handle regions which require higher resolution. The grid is represented using unstructured data that connects cells through cell faces. The details of the algorithm can be found in Singh and Shyy. ${ }^{7}$ Adaptation is performed based on the flow solution quality for effective computation as well as the interface location.

The geometry-based adaptation around interfaces allows better resolution in the near wall region and phase boundary. Near fluid interfaces, for which discontinuous flow properties smear across two layers of cells, refinement is performed to improve the resolution there to capture detailed flow structures more accurately. This fully resolved region around a fluid interface is further extended to six layers of cells to handle both the discrete Dirac function as well as a possibility for large deformation of the interface. On the other hand, solid interfaces, where interfacial dynamics are not smeared, triggers full refinement with two-cell layers from the wall. Figure 6 shows examples of geometry-based adaptation in a confined fuel tank and velocity- and temperature-solution based grid adaptation in a 2-D square natural convection case. The computational cells are dynamically refined based on the solution of the flow field, and the present implementation uses a curl based adaptation criterion for velocity, and gradient or divergence based criteria for temperature comparing cell value with a standard deviation of the whole domain.

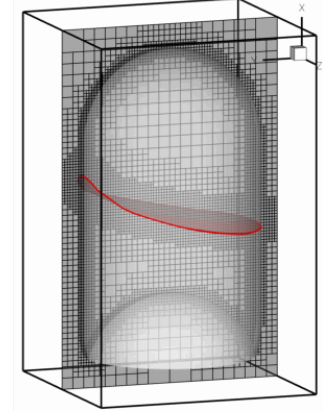

(a)

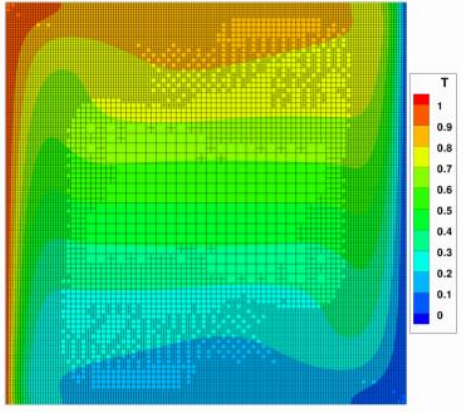

(b)

Figure 6. Adaptive grids (a) geometry-based adaptation (b) solution-based adaptation

\section{G. Mass transfer due to phase change}

Mass transfer computation is one of the key issues in the phase change process since it is related to the movement of phase boundary and the amount of latent heat in energy transfer process. In the present study, the mass transfer is computed in Eq. (18) based on the Stefan condition using the temperature gradient with discontinuous material properties for simplicity and accuracy. Where, $\mathrm{L}$ is latent heat and $\mathrm{k}$ is thermal conductivity. The interface temperature is assumed equal to the saturation temperature since it is an adequate assumption in macroscopic problems. $^{10}$

$$
\dot{\mathrm{m}}_{\mathrm{f}} \mathrm{L}=\left(\mathrm{q}_{2}-\mathrm{q}_{1}\right) \cdot \hat{\mathrm{n}}=-\left.\mathrm{k}_{2} \frac{\partial \mathrm{T}}{\partial \mathrm{n}}\right|_{2}+\left.\mathrm{k}_{1} \frac{\partial \mathrm{T}}{\partial \mathrm{n}}\right|_{1}
$$

The energy equation of Eq. (3) is solved using continuous treatment with smoothed material properties in Eqs. (4)-(7). The governing equations (1)-(2) are solved using a projection method with a staggered grid finite volume formulation. The major difference from the previous isothermal equations, the divergence of velocity is not zero around interface due to mass transfer. Shin and Juric (2002) ${ }^{11}$ developed the conservation of mass in Eq. (19), and modified Eqs. (20) for non-conservative form is implemented in the present research.

$$
\begin{gathered}
\nabla \cdot(\rho \mathbf{u})=\mathbf{u} \cdot \nabla \rho+\frac{\left(\rho_{2}-\rho_{1}\right)}{\rho_{\mathrm{f}}} \int_{\Gamma(\mathrm{t})} \dot{\mathrm{m}}_{\mathrm{f}} \delta(\mathbf{x}-\mathbf{X}) \mathrm{d} s \\
\nabla \cdot \mathbf{u}=\frac{1}{\rho}\left[\left(\rho_{2}-\rho_{1}\right) \frac{\dot{\mathrm{m}}_{\mathrm{f}}}{\rho_{\mathrm{f}}} \nabla \mathrm{I}\right]=\frac{1}{\rho}\left[\frac{\left(\rho_{2}-\rho_{1}\right)}{\rho_{\mathrm{f}}} \int_{\Gamma(\mathrm{t})} \dot{\mathrm{m}}_{\mathrm{f}} \delta(\mathbf{x}-\mathbf{X}) \mathrm{ds}\right]
\end{gathered}
$$




\section{Computational Assessment}

To highlight the performance of the present approach for moving interfacial dynamics, spacecraft fuel draining, sloshing, and surface stability cases are summarized, and validation studies for the implemented energy equation and phase change model is conducted using natural convection in a cavity and one- and two-phase Stefan problems.

\section{A. Interfacial dynamics under variable gravitational acceleration}

\section{Spacecraft fuel draining dynamics}

The dynamics of the fuel delivery at micro-gravity conditions are studied numerically and compared with experimental study conducted by Symons at zerogravity facility of NASA Lewis research center. ${ }^{16}$ Figure 7 shows the experimental and numerical configuration. The hemispherical bottomed tank with small outlet, radius of which is $1 / 10$ of tank radius $(R)$, is represented by solid interface treatment on the Stationary Cartesian grid. Non-dimensional initial liquid height, based on tank radius, is set to $3 R$. All simulations are conducted in a micro-gravitational environment with $1.5 \%$ of normal gravity, and an ellipsoidal shape that corresponds to the initial fuel volume is used for the initial interface geometry at micro-gravity since the experiment is conducted after transition time from normal gravity to micro-gravity in

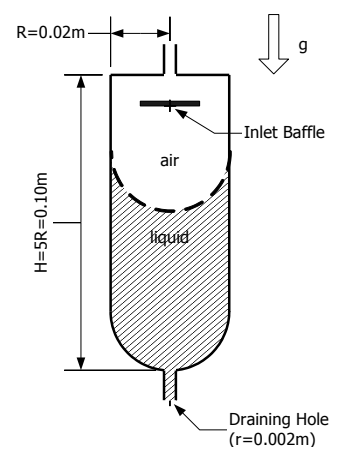

(a)

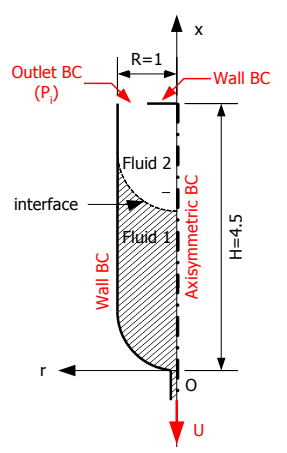

(b)

Figure 7. Geometry configuration, (a) experimental configuration, (b) computational configuration the drop tower facility on the ground.

According to Symons, the flow characteristics of the draining process can be classified into three main categories; inertia-dominated, transition, and capillary-dominated regimes. In the present study, Bond number is fixed at 5 with $\mathrm{g}=0.015 \mathrm{~g}_{0}$, and various Weber numbers are chosen from 0.1 that corresponds to draining parameter 0.01667 to 80.0 which corresponds to draining parameter 13.33 in order to verify the flow characteristics for each regime in terms of the change in sloshing waves and residual volume.

Figure 8 shows the time history of the non-dimensional height at the centerline and at the wall attachment point for the mentioned regime conditions. Contrary to the draining phenomena in a normal gravity, the fuel surface goes down with different velocity at centerline and wall varying downward speed. This results in sloshing motion repeating flat surface and elongated surface. Capillary-dominated regime is characterized by many slosh waves with small magnitudes. In this regime, the fluid goes down with almost same velocity both on the centerline and wall while maintaining its initial interface shape due to dominating capillary forces. The transition regime shows a few slosh waves with large amplitude due to competition between capillary and inertial force. In the inertia-dominated regime, the wall attachment point rarely moves while the interface at centerline moves down with a constant velocity until vapor ingestion. This regime is observed for large draining parameters and causes a larger residual volume at the time of vapor ingestion because of the almost stationary wall attachment point. The validation study is carried using a transition regime case, corresponding draining parameter $\mathrm{We} /(\mathrm{Bo}+1)=0.16$ for which the experimental guidance is available. ${ }^{16}$ The present study shows a reasonable agreement with Symons' experimental study $^{16}$ as sloshing motion and sudden vapor ingestion phenomena are captured in detail, whereas the wall contact point location is slightly different in the beginning of draining process possibly as a result of differences in the initial conditions.

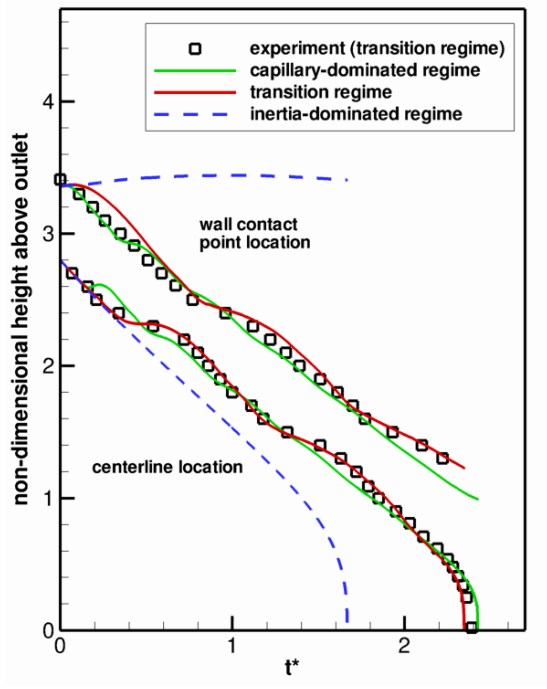

Figure 8. Non-dimensional height at the centerline and on the tank wall at capillarydominated $(\mathrm{We} /(\mathrm{Bo}+\mathbf{1})=\mathbf{0 . 0 3})$, transition $(\mathrm{We} /(\mathrm{Bo}+1)=0.3)$, and inertia-dominated regime $(\mathrm{We} /(\mathrm{Bo}+1)=13.3)$. Initial fill level is 3 tank radii. 


\section{Sloshing of cryogenic propellants in a spacecraft fuel tank}

A sloshing cryogenic propellant in a spacecraft fuel tank due to a sudden reduction of acceleration during engine shutdown is simulated. Figure 9(a) shows the configuration of the scale model of the Saturn V/S-IVB liquid hydrogen tank experimented by Toole et al. ${ }^{17}$ Their concave-shaped tank is illustrated by the triangular solid interface elements in Figure 9(b) for 3D computation on the stationary Cartesian grids. Petroleum ether and air are utilized as a substitute of liquid fuel and pressurizing gas respectively as they are in the experiment.

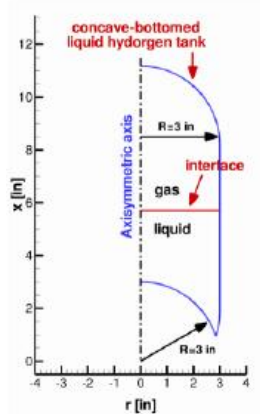

(a)

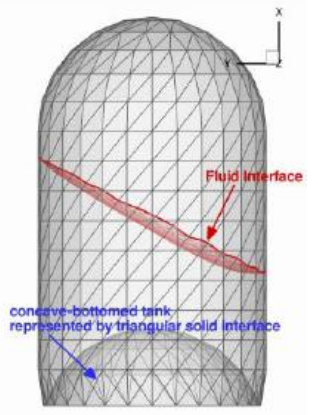

(b)

Figure 9. The computational geometry configuration of draining fuel tank with hemispherical bottom (a)axisymmetric domain, (b) 3D computational domain

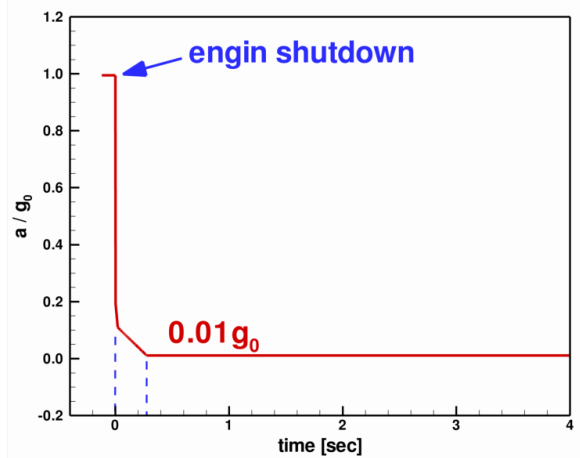

Figure 10. Acceleration history for simulation with engine shutdown at $t=0$. The acceleration decreases from normal gravity to micro-gravity.

Based on this experimental investigation, a test case with $\mathrm{Bo}=24$ and $\mathrm{Fr}=14.6$ is chosen to assess our computational modeling performance. These values correspond to an acceleration level of $0.01 \mathrm{~g}_{0}$ and maximum fluid velocity of $0.33 \mathrm{~m} / \mathrm{s}$. One of the difficulties is to match Froude number with experiment since any information on the kinetic energy at a given interface shape is not available. There is no record for the impacting force to make the initial sloshing motion, thus we pick a free surface shape from the snapshot with highest wall attachment point, then adjust the acceleration time to match the Froude number measuring the maximum velocity of a wall attachment point by trial and error.

Figure 11 shows a sloshing cryogenic propellant in a spacecraft fuel tank due to a sudden reduction of acceleration during engine shutdown. The fluid is accelerated from the initial stationary position as the potential energy is converted into kinetic energy by acceleration of normal gravity. At $t=0.00 \mathrm{sec}$, the acceleration suddenly reduced into $0.01 \mathrm{~g}_{0}$, and the high kinetic energy obtained at normal gravity is converted back into potential energy at micro-gravity. Smaller acceleration results in higher liquid fuel position to get the same potential energy level. The liquid fuel reaches and stays at the top of the tank for the time being, and then, it retracts and makes the second sloshing motion. In this simulation, the liquid fuel shows very large sloshing motions, and the center of mass changes in time up to $46 \%$ of the tank radius. The huge change of the center of mass requires excessive demands on the whole vehicle dynamics and control systems.

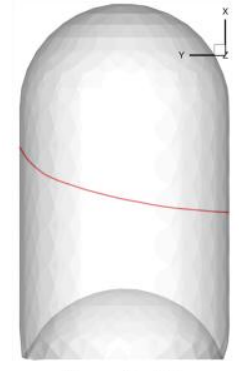

$\mathrm{t}=-0.12$

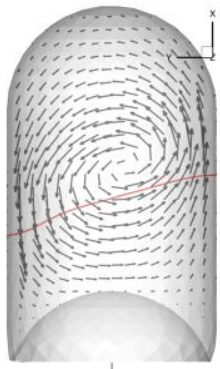

$\mathrm{t}=+0.08$

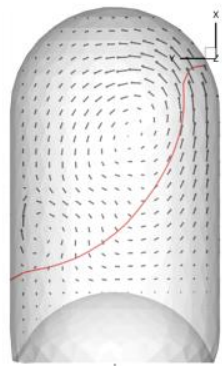

$\mathrm{t}=+0.54$

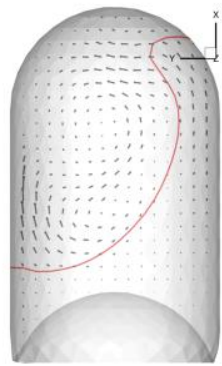

$t=+1.00$

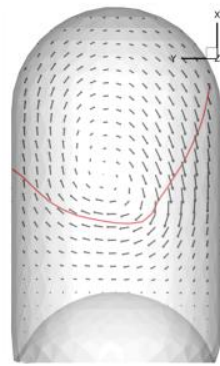

$\mathrm{t}=+2.44$

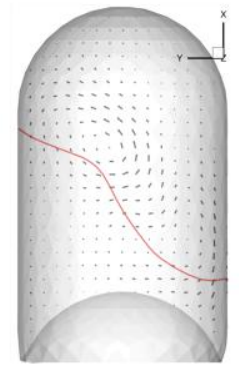

$\mathrm{t}=+3.84$

Figure 11. Simulation of sloshing fuel tank flow due to sudden reduction of acceleraton. The velocity vector and fluid surface are represented on a center-cut plane. 


\section{The liquid fuel surface stability of a spacecraft under thrust oscillation}

Thrust oscillation is one of the difficult problems in liquid and/or solid rocket designs since it has influence on the whole rocket system including astronauts' safety, and sometimes results in even rocket structure breakups. Active controls have been applied to reduce such a dangerous pogo oscillation and a kind of shock absorbers have been installed for the safety of astronauts and payloads by mitigating thrust oscillation. In this study, the influence of thrust oscillation on the liquid fuel tank is investigated. In a practical rocket system, thrust oscillation is composed of many different frequencies and magnitudes produced by different parts of rocket. ${ }^{18}$ The present study focused on the parametrically excited liquid fuel surface wave, namely Faraday wave where the vertical sinusoidal oscillation is applied to liquid/air in an open container of Figure 12.

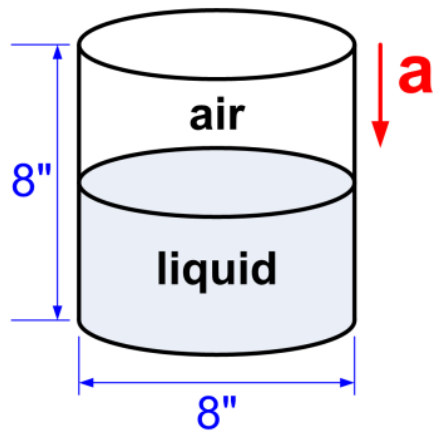

(a)

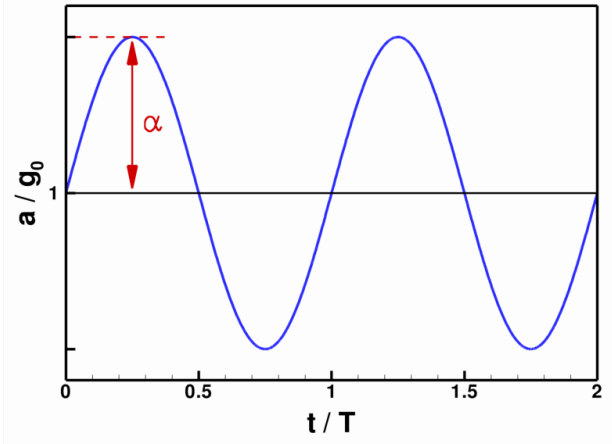

(b)

Figure 12. Geometric configuration of 3-D Faraday waves (a) 3-D configuration (b) sinusoidal acceleration

According to the dispersion relation for infinite depth periodic waves, the surface waves become unstable due to either gravitational effects or surface tension effects. It is observed that the surface wave can exhibit periodic standing waves, as well as reach aperiodic unstable states with upward jets and droplets when the acceleration exceeds a certain threshold value as the forcing oscillation magnitude increases. Figure 13 shows such aperiodic unstable states over threshold acceleration. An example of gravity wave, where gravitational effects are more crucial, at low forcing frequency $(\mathrm{f}=10 \mathrm{~Hz})$ shows a single large liquid jet at the center of axis in Figure 13(a). The transition from gravity wave into capillary wave is shown in Figure 13(b), where a large upward jet is replaced by smaller multiple jets at multiple locations. It is noted that a higher forcing frequency results in a smaller wave length, and consequently smaller multiple jets. The details can be found in Sim and Shyy (2009). ${ }^{15}$

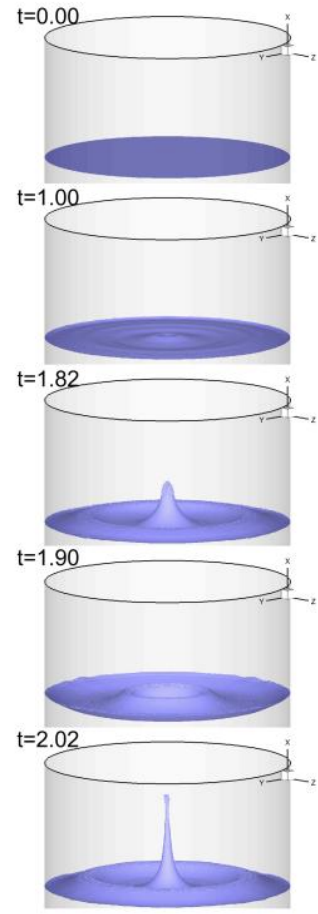

(a)

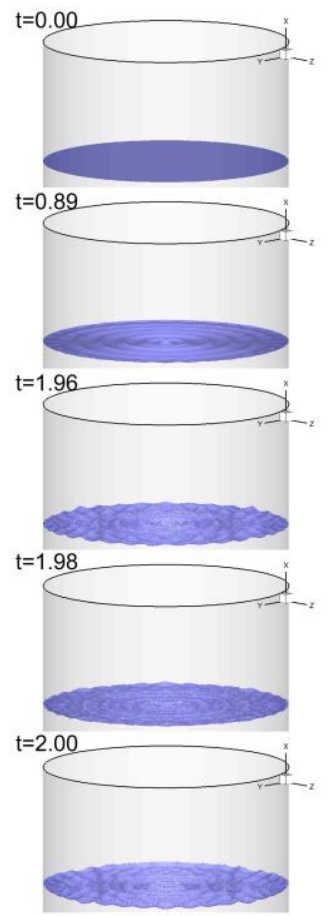

(b)

Figure 13. Comparison between gravity waves and capillary-transition waves in aperiodic surface states for water/air case. (a) $\mathrm{f}=10 \mathrm{~Hz}$ and $\alpha=0.3$ (b) $\mathrm{f}=20 \mathrm{~Hz}$ and $\alpha=0.6$ 


\section{B. Buoyancy-driven natural convection in an enclosed cavity}

The laminar natural convection flow driven by buoyancy force in a cavity is studied and compared with benchmark solution to validate the present numerical approaches on the thermal effect. Figure 14 shows the numerical configuration, where the enclosed cavity is heated differentially at $\mathrm{T}_{h}$ and $\mathrm{T}_{\mathrm{c}}$ on the vertical walls and the horizontal walls are thermally insulated. The Prandtl number $(\operatorname{Pr}=v / \alpha)$ is fixed to be 0.71 , where $v$ is kinematic viscosity and $\alpha$ is thermal diffusivity. The simulation is conducted for the Rayleigh numbers of $10^{3}, 10^{4}, 10^{5}$ and $10^{6}$, and compared with benchmark solution by de Vahl Davis. ${ }^{19}$, 20 The Rayleigh number is defined as $\mathrm{Ra}=$ $\mathrm{g} \beta \Delta \mathrm{TL}^{3} /(\alpha \nu)$, where $\mathrm{g}$ is gravitational acceleration, $\beta$ is thermal expansion, $\Delta \mathrm{T}$ is the difference of temperature between hot and cold walls, and L is the width of cavity.

An example of buoyancy-driven natural convection flow at $\mathrm{Ra}=10^{5}$ is shown in Figure 15. The flow is moving up along the hot wall on the left side, and going down along the cold wall on the right side due to buoyancy force, and these buoyancy effect makes two counterrotating eddies on the center of cavity. The present study shows second-order of accuracy due to used second order central difference scheme, and results in less than $0.1 \%$ of errors comparing with benchmark solution in Figure 16. Table 1 summarizes the errors for various variables at Rayleigh numbers of $10^{3}$ to $10^{6}$. For Rayleigh numbers less than $10^{5}$, the error is less than $1 \%$ and shows good agreement, but the errors tend to increase as Rayleigh number increases. The Nusselt number usually shows larger errors due to the effect of post processing methods.

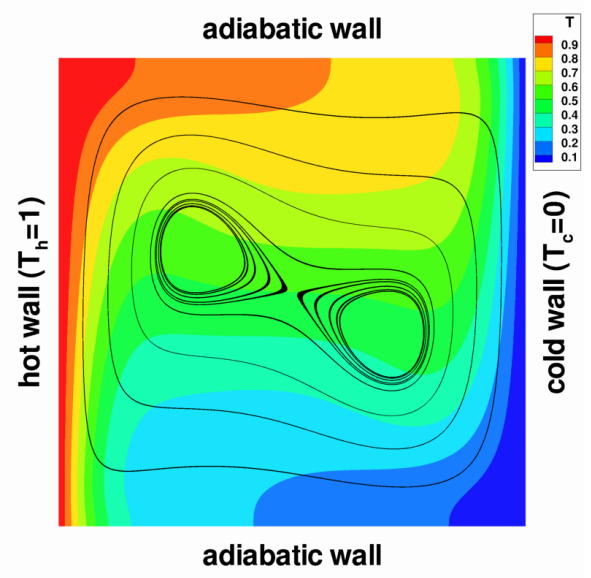

Figure 15. Temperature contour and streamlines at $\mathbf{R a}=10^{5}$.

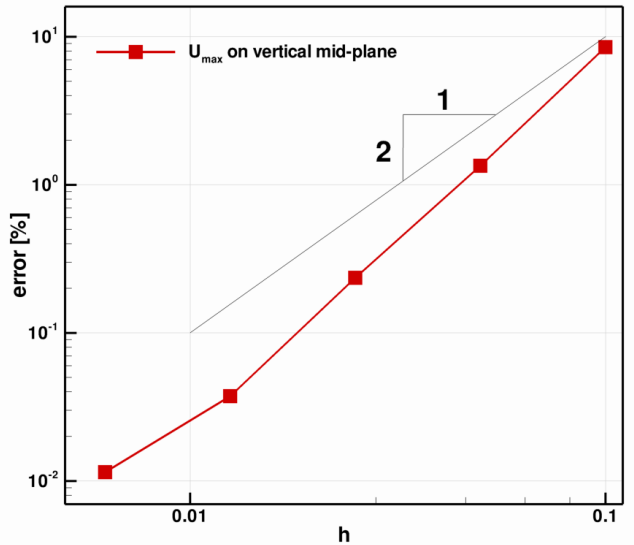

Figure 16. Grid convergence test for $U_{\max }$ on vertical mid-plane based on the benchmark solution.

The flow features of natural convection in a 2-D square cavity is investigated changing Rayleigh number in Figure 17, where the boundary layer grows along the wall and the flow fields becomes more asymmetric as Rayleigh number increases. For $\mathrm{Ra}=10^{3}$ and $10^{4}$, one large recirculation zone is created at the center of the enclosed cavity as the flow is moving up along left side of hot wall and going down along the right side of cold wall. The one recirculation zone is divided into two counter-rotating smaller eddies at $\mathrm{Ra}=10^{5}$. These counter-rotating eddies move to corners of top-left and bottom-right and another eddie is created between them as Rayleigh number reaches to $10^{6}$. 
Table 1. The comparison between present study and benchmark solution

\begin{tabular}{|c|c|c|c|c|}
\hline & \multicolumn{4}{|c|}{ Errors [\%] on 81x81 grid } \\
\hline $\mathrm{Ra}$ & $10^{3}$ & $10^{4}$ & $10^{5}$ & $10^{6}$ \\
\hline $\mathrm{U}_{\max }$ & 0.06 & 0.09 & 0.04 & 0.79 \\
\hline $\mathrm{V}_{\max }$ & 0.08 & 0.14 & 0.46 & 0.81 \\
\hline $\mathrm{Nu}$ & 0.00 & 0.09 & 0.27 & 1.40 \\
\hline $\mathrm{Nu}_{1 / 2}$ & 0.00 & 0.00 & 0.35 & 1.08 \\
\hline $\mathrm{Nu}_{0}$ & 0.09 & 0.45 & 0.71 & 1.62 \\
\hline $\mathrm{Nu}_{\max }$ & 0.20 & 0.43 & 1.34 & 1.19 \\
\hline $\mathrm{Nu}_{\min }$ & 0.15 & 0.34 & 0.69 & 2.63 \\
\hline
\end{tabular}

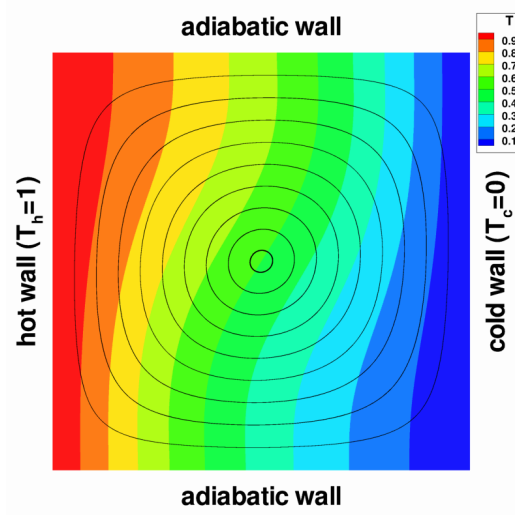

(a) $\mathrm{Ra}=10^{3}$

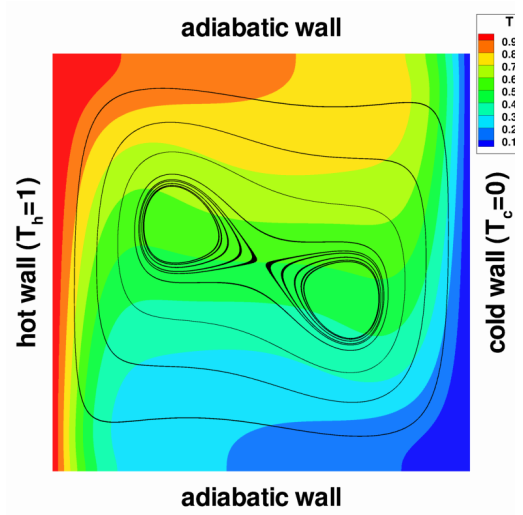

(c) $\mathrm{Ra}=10^{5}$

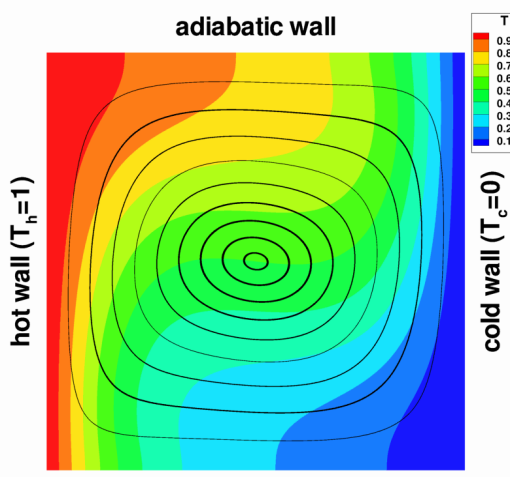

adiabatic wall

(b) $\mathrm{Ra}=10^{4}$

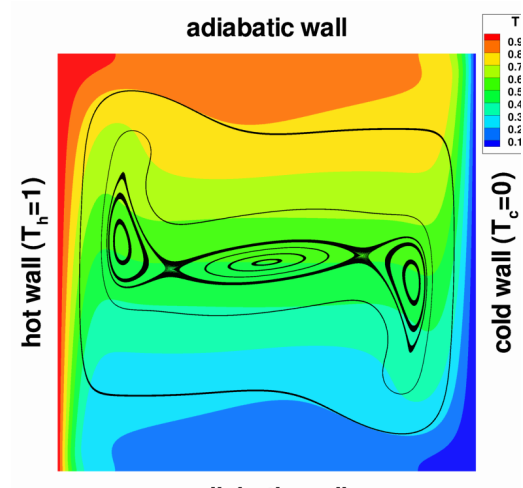

adiabatic wall

(d) $\mathrm{Ra}=10^{6}$

Figure 17. The effect of Rayleigh number in natural convection of a 2-D square cavity; temperature contour and streamlines (a) $\operatorname{Ra}=10^{3}$ (b) $\operatorname{Ra}=10^{4}$ (c) $\operatorname{Ra}=10^{5}$ (d) $\mathrm{Ra}=10^{6}$

The detailed profiles of velocities and temperature and Nusselt number distribution are plotted in Figure 18. The temperature distribution shows rapid changes on the hot and cold vertical walls as Rayleigh number increases, which, in turn, results in corresponding velocity profiles. The Nusselt number distribution along vertical walls shows this more clearly. Nusselt number, thus heat transfer increases as Rayleigh number increases, and it shows asymmetric heat transfer; most of heat is transferred from bottom on the hot wall of left side, and from top on the cold wall of right side.

The capability of 3-D computation is tested using natural convection in a cubic with two different boundary conditions. Figure 19(a) shows the common numerical configuration with hot and cold walls on x-planes, and cases with 4 adiabatic walls or 4 conducting walls heated by $T=x$ are shown in Figure 19(b) and (c). 


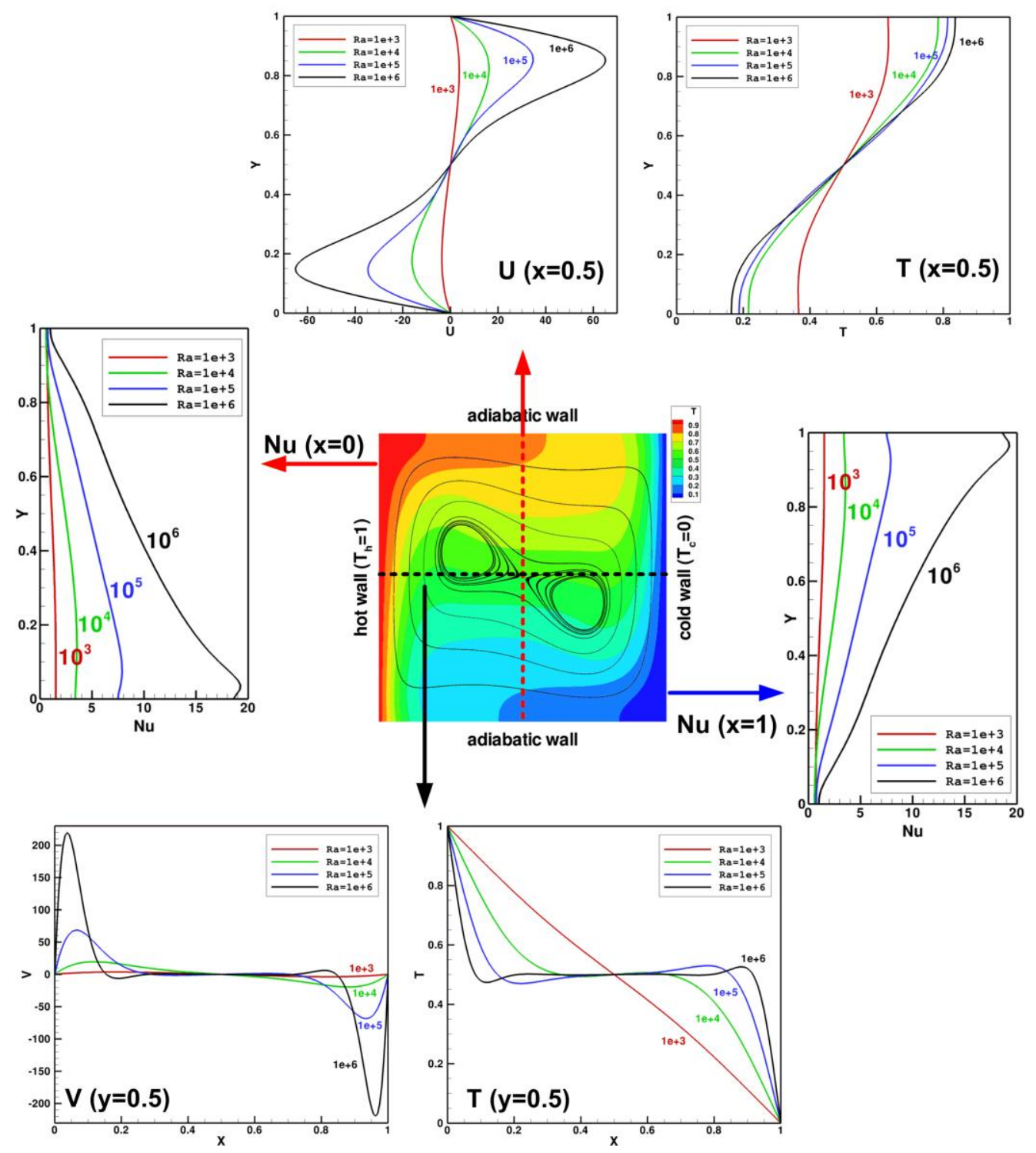

Figure 18. The effect of Rayleigh number in natural convection of a 2-D square cavity from $R a=10^{3}$ to $10^{6}$; velocity, temperature and Nusselt number

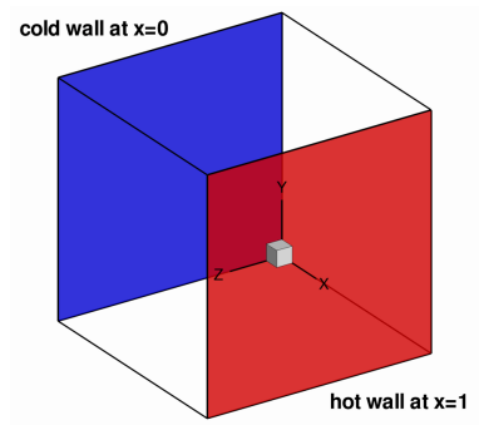

(a)

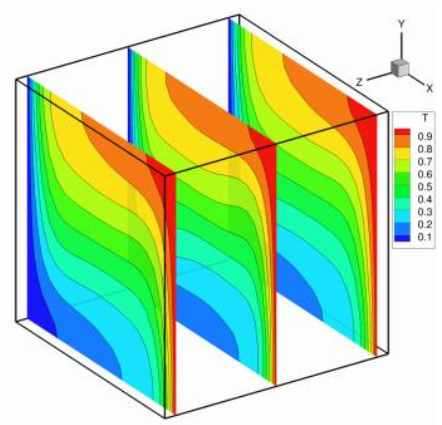

(b)

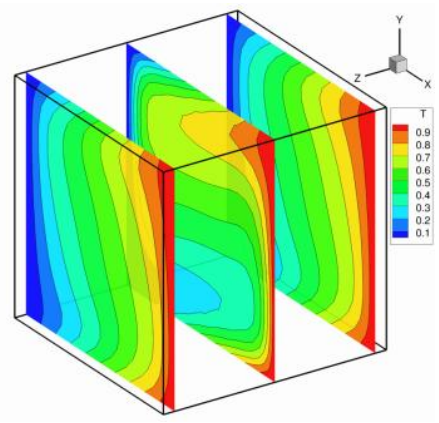

(c)

Figure 19. Natural convection in a 3-D cavity (a) numerical configuration (b) case with 4 adiabatic walls (c) case with 4 conducting walls heated by $T=x$ on $y$ - and z-plane. 


\section{The validation of phase change computation}

A one dimensional phase change test problem is performed to evaluate the present computational approach for phase change. One-phase and two-phase Stefan problem is validated and compared with theoretical solution. ${ }^{21}$

Figure 20 shows the numerical configuration for these Stefan problem on a semi-infinite slab, where the left wall was maintained at a constant temperature $T_{0}$ with the interface location, $X(t)$, denoted by separating the vapor and liquid phases on the left and right sides respectively. The one-phase Stefan problem in Figure 20(a) is the simplest explicitly solvable phase change problem, where the phase 2 is assumed staying at the saturation temperature same to the temperature at the interface location, and thus, the solution is independent on the phase 2. However, this assumption is unrealistic scenario in a real world. In the two-phase Stefan problem of Figure 20(b), the initial temperature of phase 2 is a certain value, $T_{\infty}$, but varies in time due to heat transfer from phase 1 . These one- and two-phase Stefan problems are summarized in Table 2.

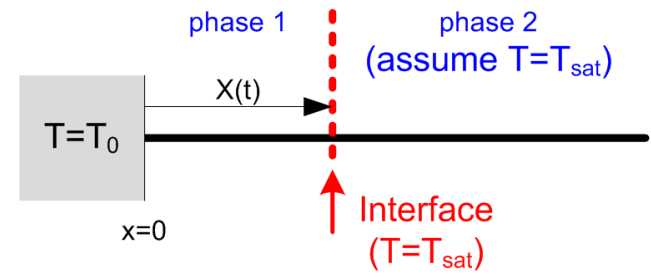

(a)

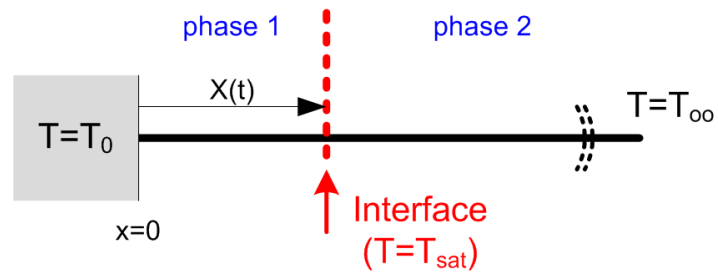

(b)

Figure 20. Numerical configuration of one dimensional phase change test problem (a) one-phase Stefan problem (b) two-phase Stefan problem

Table 2. One dimensional Stefan problem

\begin{tabular}{|c|l|l|}
\hline & \multicolumn{1}{|c|}{ One-phase Stefan problem } & \multicolumn{1}{|c|}{ Two-phase Stefan problem } \\
\hline $\begin{array}{c}\text { Interface } \\
\text { temperature }\end{array}$ & $\mathrm{T}(\mathrm{X}(\mathrm{t}), \mathrm{t})=\mathrm{T}_{\text {sat }}, \quad(\mathrm{t}>0)$ & $\mathrm{T}(\mathrm{X}(\mathrm{t}), \mathrm{t})=\mathrm{T}_{\text {sat }}, \quad(\mathrm{t}>0)$ \\
\hline Stefan condition & $\rho \mathrm{LX}(\mathrm{t})=-\mathrm{k}_{1} \mathrm{~T}_{\mathrm{x}}(\mathrm{X}(\mathrm{t}), \mathrm{t}), \quad(\mathrm{t}>0)$ & $\rho \mathrm{LX}(\mathrm{t})=-\mathrm{k}_{1} \mathrm{~T}_{\mathrm{x}}\left(\mathrm{X}(\mathrm{t})^{-}, \mathrm{t}\right)+\mathrm{k}_{2} \mathrm{~T}_{\mathrm{x}}\left(\mathrm{X}(\mathrm{t})^{+}, \mathrm{t}\right)$ \\
\hline \multirow{2}{*}{ Initial condition } & $\mathrm{T}(\mathrm{x}, 0)=\mathrm{T}_{\mathrm{sat}}, \quad(\mathrm{x}>0)$ & $\mathrm{T}(\mathrm{x}, 0)=\mathrm{T}_{\infty}<\mathrm{T}_{\mathrm{sat}}, \quad(\mathrm{x}>0)$ \\
\cline { 2 - 3 } & $\mathrm{X}(0)=0$ & $\mathrm{X}(0)=0$ \\
\hline \multirow{2}{*}{ Boundary condition } & $\mathrm{T}(0, \mathrm{t})=\mathrm{T}_{0}>\mathrm{T}_{\text {sat }}, \quad(\mathrm{t}>0)$ & $\mathrm{T}(0, \mathrm{t})=\mathrm{T}_{0}>\mathrm{T}_{\text {sat }}, \quad(\mathrm{t}>0)$ \\
\cline { 2 - 3 } & $\mathrm{T}(\mathrm{x}, \mathrm{t})=\mathrm{T}_{\text {sat }}, \quad(\mathrm{x} \geq \mathrm{X}(\mathrm{t}), \mathrm{t}>0)$ & $\lim _{\mathrm{x} \rightarrow \infty} \mathrm{T}(\mathrm{x}, \mathrm{t})=\mathrm{T}_{\infty}, \quad(\mathrm{t}>0)$ \\
\hline
\end{tabular}

The theoretical interface location and temperature distribution for one-phase Stefan problem are given by Eqs. (21)-(23) where $\alpha$ and $C$ are thermal diffusivity and heat capacity of the phase 1 and $\mathrm{L}$ is the latent heat of phase change such as evaporation or melting. Parameter $\lambda$ is obtained by solving the transcendental Eq. (23).

$$
\begin{gathered}
\mathrm{X}(\mathrm{t})=2 \lambda \sqrt{\alpha \mathrm{t}} \\
\mathrm{T}(\mathrm{x}, \mathrm{t})=\mathrm{T}_{0}-\left(\frac{\mathrm{T}_{0}-\mathrm{T}_{\text {sat }}}{\operatorname{erf}(\lambda)}\right) \operatorname{erf}\left(\frac{\mathrm{x}}{2 \sqrt{\alpha \mathrm{t}}}\right), \quad \text { (for phase 1) } \\
\lambda \exp \left(\lambda^{2}\right) \operatorname{erf}(\lambda)=\mathrm{C}\left(\frac{\mathrm{T}_{0}-\mathrm{T}_{\text {sat }}}{\mathrm{L} \sqrt{\pi}}\right)
\end{gathered}
$$

The computations are started with the initial interface location at $\mathrm{X}(\mathrm{t})=0.1$ using the material properties in Table 3. The parameter $\lambda=0.62006263$ is used based on the solution of transcendental equation for the chosen fluid properties. The theoretical temperature distribution at the time corresponding initial interface location is imposed as the initial condition of the present numerical study with computational domain from $x=0$ to $x=1$ using 100 grid cells. The interface location in time due to phase change is shown in Figure 21(a), where the interface moves fast in the 
beginning and slows down as time goes since the temperature gradient become smaller as the interface goes far from the hot temperature at $\mathrm{x}=0$. The present study shows good agreement with analytical solution in the interface location and temperature distribution at $\mathrm{t}=0.2$.

Table 3. parameters for one-dimensional phase change computation.

\begin{tabular}{|c|c|c|}
\hline Parameter & Case 1 & Case 2 \\
\hline $\mathrm{T}_{0}$ & 1.0 & 1.0 \\
\hline $\mathrm{T}_{\mathrm{sat}}$ & 0.0 & 0.0 \\
\hline $\mathrm{T}_{\infty}$ & & -1.0 \\
\hline density $(\rho)$ & 1.0 & 1.0 \\
\hline heat capacity $(\mathrm{C})$ & 1.0 & 1.0 \\
\hline thermal conductivity $\left(\mathrm{K}_{1}\right)$ & 1.0 & 1.0 \\
\hline thermal conductivity $\left(\mathrm{K}_{2}\right)$ & 1.0 & $0.1 / 1.0 / 10.0$ \\
\hline
\end{tabular}

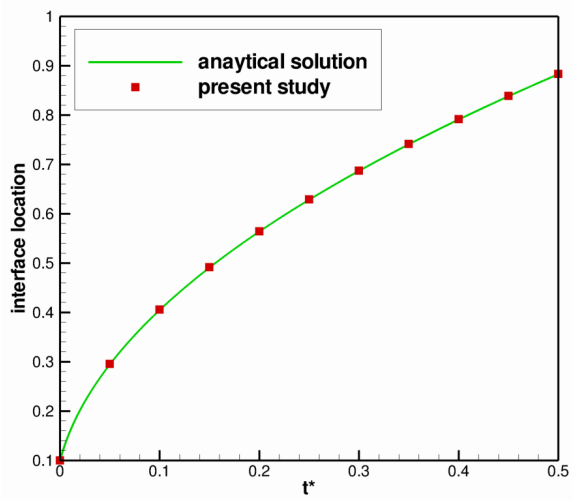

(a)

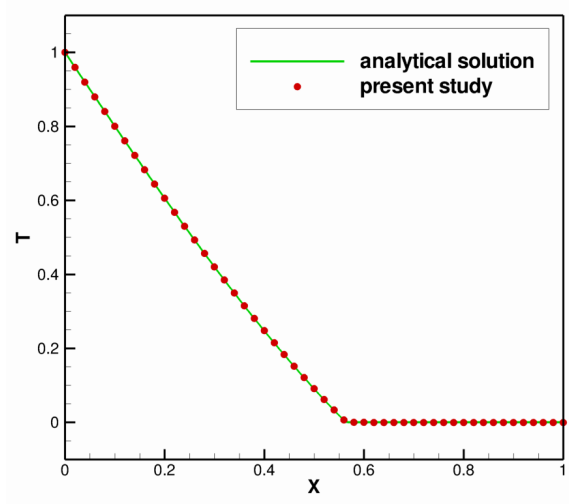

(b)

Figure 21. Comparison of present numerical study and analytical solution for one-phase Stefan problem in Table 3. (a) Interface location and (b) temperature profile at time $=0.2$.

In the two-phase Stefan problem, the temperature of phase 2 is different from saturation temperature, and varies in time due to heat transfer from phase 1, and thus, the solution is dependent on both phase 1 and 2 in Eqs. (24)-(27).

$$
\begin{gathered}
\mathrm{X}(\mathrm{t})=2 \lambda \sqrt{\alpha \mathrm{t}} \\
\mathrm{T}(\mathrm{x}, \mathrm{t})=\mathrm{T}_{0}-\left(\frac{\mathrm{T}_{0}-\mathrm{T}_{\text {sat }}}{\operatorname{erf}(\lambda)}\right) \operatorname{erf}\left(\frac{\mathrm{x}}{2 \sqrt{\alpha_{1} \mathrm{t}}}\right), \quad \text { (for phase 1) } \\
\mathrm{T}(\mathrm{x}, \mathrm{t})=\mathrm{T}_{\infty}+\left(\frac{\mathrm{T}_{\mathrm{sat}}-\mathrm{T}_{\infty}}{\operatorname{erfc}\left(\lambda \sqrt{\alpha_{1} / \alpha_{2}}\right)}\right) \operatorname{erfc}\left(\frac{\mathrm{x}}{2 \sqrt{\alpha_{2} \mathrm{t}}}\right), \quad \text { (for phase 2) } \\
\lambda=\frac{\mathrm{C}_{1}\left(\mathrm{~T}_{0}-\mathrm{T}_{\mathrm{sat}}\right)}{\mathrm{L} \sqrt{\pi} \exp \left(\lambda^{2}\right) \operatorname{erf}(\lambda)}-\frac{\mathrm{C}_{2}\left(\mathrm{~T}_{\mathrm{sat}}-\mathrm{T}_{\infty}\right)}{\mathrm{L} \sqrt{\pi} \exp \left(\left(\lambda \sqrt{\alpha_{1} / \alpha_{2}}\right)^{2}\right) \operatorname{erfc}\left(\lambda \sqrt{\alpha_{1} / \alpha_{2}}\right)}
\end{gathered}
$$

The two-phase Stefan problem is simulated varying thermal conductivity ratio from 0.1 to 10 . It was known that continuous interface treatment has some errors in simulating thermal effect. However, Ferziger showed the error is due to incorrect smoothed averaging of material properties in the stationary heat conduction problem with no phase 
change. ${ }^{14}$ In the present study, the thermal conductivity is averaged using in the Eqs. (4)-(7), and it results in good agreement with analytical solution even for phase change cases. Figure 22 shows the interface location and temperature distribution for different thermal conductivity ratio from 0.1 to 10 , and it matches very well with analytical solution. The different temperature profile for each case is shown in Figure 22(b), where small thermal conductivity in phase 2 produces large temperature gradient, and results in fast movement of interface due to large mass transfer.

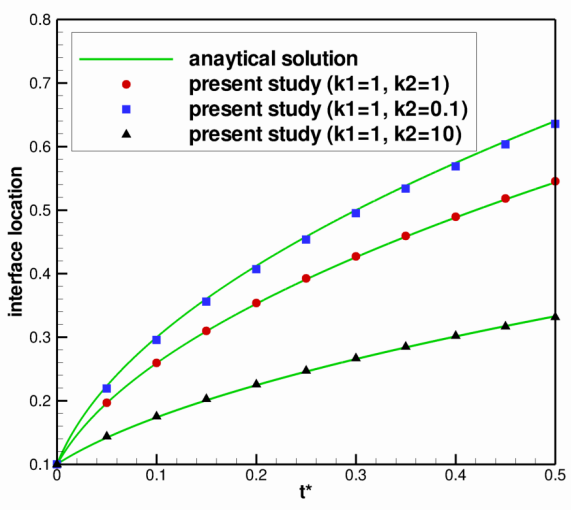

(a)

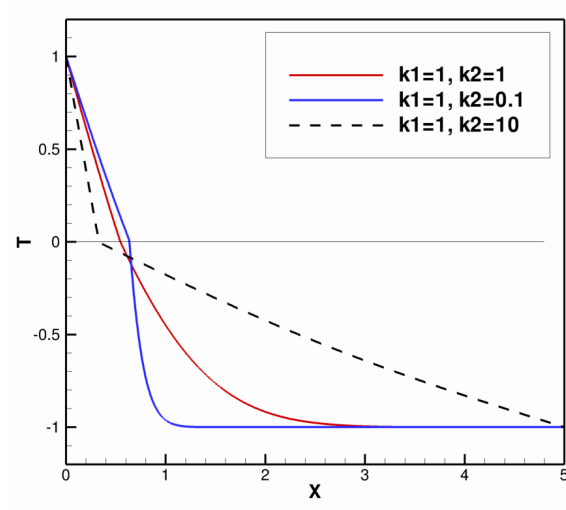

(b)

Figure 22. Comparison of present numerical study and analytical solution for two-phase Stefan problem in Table 3. (a) Interface location and (b) temperature profile at time $=0.2$.

\section{Summary and Conclusions}

In this paper, we report recent efforts in developing a multi-scale three-dimensional adaptive Cartesian grid method for multiphase flow computation. The method utilizes marker-coded triangulated surface for both tracking interfaces separating fluid constituents and arbitrarily shaped solid geometries. The markers can move as required by physics and/or prescribed boundary conditions. The governing equations are solved on the Eulerian, Cartesian grid with automated local grid adaptation to capture the fluid flow and thermal structures with desirable resolution. The model can also handle phase change. Numerous test cases have been adopted to evaluate the performance of the modeling and computational capabilities of the present approach, including:

(1) Liquid fuel draining dynamics at micro-gravity,

(2) Sloshing motion and dynamics of cryogenic propellant in a spacecraft fuel tank due to a sudden reduction of acceleration,

(3) Liquid fuel surface stability under vertical sinusoidal thrust oscillation,

(4) Natural convection in an enclosed cavity due to buoyancy force,

(5) one- and two-phase Stefan phase change problem.

We are in the process of further refining and assessing the computational techniques along with detailed code verification and validation exercises for flows around complex-shaped solid and interfacial boundaries, as well as systems involving large property jumps.

\section{Acknowledgments}

The work reported in this paper has been partially supported by NASA Constellation University Institutes Program (CUIP), Claudia Meyer and Jeff Rybak program managers. We have benefited from the discussion with John Peugeot and Jeff West of NASA Marshall Space Flight Center. 


\section{References}

${ }^{1}$ Shyy, W., Udaykumar, H.S., Rao, M.M., and Smith, R.W., Computational Fluid Dynamics with Moving Boundaries. 1996, Philadelphia: Taylor \& Francis.

${ }^{2}$ Osher, S. and Fedkiw, R., Level Set Methods and Dynamic Implicit Surfaces. 2002, New York: Springer.

${ }^{3}$ Sethian, J.A. and Smereka, P., "Levet Set Methods for Fluid Interfaces," Annual Review of Fluid Mechanics, 2003. 35(1): p. 341-372.

${ }^{4}$ Tryggvason, G., Bunner, B., Esmaeeli, A., Al-Rawahi, N., Tauber, W., Han, J., Jan, Y.J., Juric, D., and Nas, S., "A fronttracking method for the computations of multiphase flow," Journal of Computational Physics, 2001. 169(2): p. 708-759.

${ }^{5}$ Prosperetti, A. and Tryggvason, G., Computational Methods for Multiphase Flow. 2007, New York: Cambridge University Press.

${ }^{6}$ Peskin, C.S., "The immersed boundary method," Acta Numerica, 2003. 11: p. 479-517.

${ }^{7}$ Singh, R. and Shyy, W., "Three-dimensional adaptive Cartesian grid method with conservative interface restructuring and reconstruction," Journal of Computational Physics, 2007. 224(1): p. 150-167.

${ }^{8}$ Mittal, R., Dong, H., Bozkurttas, M., Najjar, F.M., Vargas, A., and Loebbecke, A.v., "A versatile sharp interface immersed boundary method for incompressible flows with complex boundaries," J. Comput. Phys., 2008. 227(10): p. 4825-4852.

${ }^{9}$ Uzgoren, E., Sim, J., and Shyy, W., "Marker-based, 3-D adaptive Cartesian grid method for multiphase flow around irregular geometries.," Communications in Computational Physics, 2009. 5(1): p. 1-41.

${ }^{10}$ Juric, D. and Tryggvason, G., "Computations of boiling flows," Int. J. Multiphase Flow, 1998. 24(3): p. 387-410.

${ }^{11}$ Shin, S. and Juric, D., "Modeling three-dimensional multiphase flow using a level contour reconstruction method for front tracking without connectivity," Journal of Computational Physics, 2002. 180(2): p. 427-470.

${ }^{12}$ Luo, X.-Y., Ni, M.-J., Ying, A., and Abdou, M.A., "Numerical Modeling for Multiphase Incompressible Flow with Phase Change," Numerical Heat Transfer, Part B: Fundamentals, 2005. 48(5): p. 425 - 444.

${ }^{13}$ Morgan, N.R., A NEW LIQUID-VAPOR PHASE TRANSITION TECHNIQUE FOR THE LEVEL SET METHOD, in Mechanical Engineering. 2005, Georgia Institute of Technology: Atlanta. p. 104.

${ }^{14}$ Ferziger, J.H., "Interfacial transfer in Tryggvason's method," International Journal for Numerical Methods in Fluids, 2003. 41(5): p. 551-560.

${ }^{15}$ Sim, J. and Shyy, W. 3-D Adaptive Eulerian-Lagrangian Method for Gravity-and Capillarity-Induced Flows. in 47th AIAA Aerospace Science Meeting. 2009. Orlando, FL.

${ }^{16}$ Symons, E.P., "Draining Characteristics of Hemispherically Bottomed Cylinders in a Low-Gravity Environment," 1978.

${ }^{17}$ Toole, L.E. and Hastings, L.J., "An Experimental Study of the Behavior of a Sloshing Liquid Subjected to a Sudden Reduction in Acceleration," NASA TM X-53755, 1968.

${ }^{18}$ Yang, V. and Andersen, W.E., eds. Liquid Rocket Engine Combustion Instability. 1995, AIAA.

${ }^{19}$ Davis, G.D.V., "Natural convection of air in a square cavity: A bench mark numerical solution," International Journal for Numerical Methods in Fluids, 1983. 3(3): p. 249-264.

${ }^{20}$ Davis, G.D.V. and Jones, I.P., "Natural convection in a square cavity: A comparison exercise," International Journal for Numerical Methods in Fluids, 1983. 3(3): p. 227-248.

${ }^{21}$ Alexiades, V. and Solomon, A.D., Mathematical Modeling of Melting and Freezing Processes. 1993: Taylor \& Francis. 